\title{
The ties that bind what is known to the recall of what is new
}

\author{
DOUGLAS L. NELSON and NAN ZHANG \\ University of South Florida, Tampa, Florida
}

\begin{abstract}
Cued recall success varies with what people know and with what they do during an episode. This paper focuses on prior knowledge and disentangles the relative effects of 10 features of words and their relationships on cued recall. Results are reported for correlational and multiple regression analyses of data obtained from free association norms and from 29 experiments. The 10 features were only weakly correlated with each other in the norms and, with notable exceptions, in the experiments. The regression analysis indicated that forward cue-to-target strength explained the most variance, followed by backward target-to-cue strength. Target connectivity and set size explained the next most variance, along with mediated cue-to-target strength. Finally, frequency, concreteness, shared associate strength, and cue set size also contributed significantly to recall. Taken together, indices of prior word knowledge explain $49 \%$ of the recall variance. Theoretically driven equations that use free association to predict cued recall were also evaluated. Each equation was designed to condense multiple indices of word interconnectivity into a single predictor.
\end{abstract}

Few memory researchers would argue with the proposition that recall involves an interaction between what is known and what is new. Early work on free recall (Deese, 1965; Jenkins \& Russell, 1952) and cued recall (Bahrick, 1970 ) showed that memory is facilitated in each of these tasks by preexisting connections among the words. More recent work indicates that such connections can facilitate implicit as well as explicit recall (e.g., Nelson, Schreiber, \& Holley, 1992), can inhibit as well as facilitate performance (M. C. Anderson \& Spellman, 1995), and can produce false memories (McEvoy, Nelson, \& Komatsu, 1999; Roediger \& McDermott, 1993). Studies concerning the interaction between known and new information often involve a two-step research process. Free association data are used as a yardstick for assessing what is known, and recent study in an experimentally defined episode is used to determine what is new. When preexisting knowledge exerts an influence during a current episode, researchers draw conclusions about the role of prior experience in memory. Of course, known information comes in many forms, and one purpose of this paper is to separate the relative effects of a number of different word features known to affect cued recall. The second purpose is to develop and evaluate measurement algorithms designed to reduce multiple word-to-word connections to single-value predictors of cued recall.

This research was supported by Grant MH16360 from the National Institute of Mental Health to D.L.N. The authors thank Takako Komatsu for her help in organizing the data and Cathy McEvoy for her comments on an earlier draft. Correspondence concerning this article should be addressed to D. L. Nelson, Department of Psychology, University of South Florida, Tampa, FL 33620-8200 (e-mail: nelson@)] luna.cas.usf.edu).
In one exemplar of the two-step approach, subjects study individually presented words or targets and are then presented with other words as retrieval cues for prompting target recall (see Nelson, McKinney, Gee, \& Janczura, 1998, and Nelson, Schreiber, \& McEvoy, 1992, for reviews). The test cues are called extralist cues because they are unavailable during the study trial and cannot be directly encoded along with their to-be-recalled targets. This feature is important because subjects are forced to rely on preexisting connections during retrieval, making it an ideal task for investigating the interaction between known and new information. The mutable nature of this task represents another useful feature. In addition to manipulating the features of the studied targets and cues, the encoding and retrieval conditions can be varied independently. During either or both phases, subjects can be implicitly or explicitly oriented, they can be focused on different levels of stimulus information, context can be varied, and in short, a host of experimental variations known to affect cued recall can be varied. Given that the study trial normally takes about $1 \mathrm{~min}$ and the test trial another 2$4 \mathrm{~min}$, the extralist cuing task is akin to a semantic priming task with longer delays and a stronger episodic component.

Results obtained in the extralist cuing paradigm indicate that preexisting features contribute substantially to cued recall by influencing what is activated about to-beremembered target words during study and by influencing the effectiveness of cues presented as retrieval aids during testing (Nelson et al., 1998; Nelson \& Schreiber, 1992; Nelson, Schreiber, \& McEvoy, 1992; Nelson \& Xu, 1995). Recall is more likely when targets have fewer preexisting associates, when more of these associates are connected to each other and back to their targets, and when they are high in concreteness and low in printed frequency. Test 
cues are more effective when they are connected to fewer associates, one of which is the target. Recall is also more likely when test cues are more strongly related to their targets (the forward connection), when targets are more strongly related to their cues (the backward connection), when cues and targets share mediated connections (e.g., apple-tree-orange), and even when they share associates (e.g., both apple and orange produce fruit as an associate). Such results are robust and occur under a variety of encoding and retrieval conditions, but what is more important, they indicate that what subjects have learned about words outside the episode contributes substantially to recall within the episode.

This work is similar to other work on the role of preexisting information in that it relies on assessing prior knowledge - in this case, lexical knowledge - and then attempts to manipulate that knowledge in the context of a controlled episodic experience. The fact that word attributes, such as number of associates (set size), influence recall validates the free association yardstick as a useful index of what people know when they have been exposed to similar cultures. If the yardstick was invalid or unreliable, there would be no reason to expect that the features indexed by this method would have systematic effects (Nelson, McEvoy, \& Dennis, 2000). For example, if free association could not be used to estimate reliably how many strong associates are linked to a given word and if such associative information was not generally shared, this attribute should fail to have consistent effects on recall. The number of associates linked to a given word and other word features indexed by free association could be regarded as just another collection of random variables that contribute to error.

Despite the apparent success of this work on prior knowledge, there is an important drawback. Such work relies on indexing features in free association in order to predict memory performance in recall and recognition. Although the features are manipulated under carefully controlled conditions, they may not be independent. As far as conclusions about preexisting representations are concerned, the research is as correlational as it is manipulational, and it is legitimate to ask whether the ostensible effects produced by any given feature are direct or are produced through an association with a correlated attribute. This problem represents the classic dilemma of working with materials variables, and researchers can solve the problem in one of three ways. First, the potential role of prior knowledge can be treated as random noise and then ignored in research on the effects of variables that can be directly manipulated during the experimental episode. One problem with this approach is that evolving experimental paradigms and theories essentially treat subjects as blank tablets and ignore the potential influence of prior information altogether. Second, prior knowledge can be instilled under carefully controlled conditions, and its influence on performance can be directly studied. This approach represents the strongest tradition in experimental science, but it raises legitimate questions about ecological validity. Finally, in the measure-then-manipulate tradition, prior knowledge can be assessed in one task, and this information can be used to predict and constrain performance in another. All of our work on cued recall has been of this type, but as was noted, such work suffers from the possibility of correlated attributes that eventually must be disentangled.

This paper has two specific aims. The first and main aim is to address the problem of correlated features directly (see Rubin \& Friendly, 1986). We examined correlations between features indexed by free association in a normative database involving $58,000+$ word pairs (Nelson, McEvoy, \& Schreiber, 1999) and applied both correlation and multiple regression procedures to an experimental database for extralist cued recall involving $2,000+$ pairs and approximately 1,000 subjects. The rationale driving these analyses assumed that low correlations among word features in each database would indicate that they can be regarded as functionally independent. Alternatively, the presence of natural correlations in the normative database would suggest dependence among the features and would forecast a potential for confoundings in the experimental database.

Natural relationships among the features of words can be reduced or eliminated during the list-building process by holding these features constant across conditions. For example, when interest is focused on investigating the influence of forward strength between the test cue and the target, pairs are not selected at random, but with a view toward representing this information as a variable. Other features known to affect recall are controlled (e.g., word frequency). Attempts to manipulate some features while controlling others is a complex process in which many constraints are applied, and although such constraints may eliminate some confoundings, they may introduce others as a result of the questionable assumption that what is not controlled cancels out as random noise. The use of multiple lists in any single experiment should alleviate such confoundings, but this procedure provides no guarantees, particularly against natural relationships. What is more, in long-term research projects, features left to vary randomly early in the research only come to be controlled later in the research (e.g., Nelson, Schreiber, \& Xu, 1999). Hence, we thought it would be important to apply multiple regression procedures to the results of experiments on extralist cued recall conducted under similar conditions in the last 10 years in our laboratory. Such procedures can be used to determine whether a particular feature had an effect on probability of cued recall that is above and beyond any effects produced by its correlation with other features.

The second aim of this paper was to evaluate measurement algorithms designed to reduce multiple indices of word-to-word connection strength to single predictors. Research on cued recall indicates that many different types of preexisting connections bind words together to influence success. Some of these connections concern the associative organization of related words activated by a studied target word, and others concern the various types of connections that link this target with its test cue. Rather 
Table 1

Definitions of Features

\begin{tabular}{|c|c|}
\hline Feature & Definition \\
\hline Set size & $\begin{array}{l}\text { Number of associates of a word produced by two or more subjects in a } \\
\text { discrete free association task. }\end{array}$ \\
\hline Frequency & Printed frequency of occurrence from Kučera and Francis (1967). \\
\hline Concreteness & Ratings of word concreteness on a 1-7 scale. \\
\hline Connectivity & Mean number of connections among the associates of a target. \\
\hline Resonance & Probability that an associate of the target produces it in free association. \\
\hline Forward strength & Probability that a test cue produces its target in free association. \\
\hline Backward strength & Probability that a target produces its test cue in free association. \\
\hline $\begin{array}{l}\text { Shared associate } \\
\text { strength }\end{array}$ & $\begin{array}{l}\text { Strength of a test cue and target when connected to a third word. } \\
\text { Calculated by multiplying the probability that the test cue produces Word } \\
\mathrm{X} \text { by the probability that the target produces Word } \mathrm{X} \text { and summing the } \\
\text { results when more than a single shared associate is involved. }\end{array}$ \\
\hline Mediated strength & $\begin{array}{l}\text { Strength of a test cue and target when connected through a third word. } \\
\text { Calculated by multiplying the probability that the test cue produces Word } \\
\mathrm{X} \text { by the probability that Word } \mathrm{X} \text { produces the target and summing the } \\
\text { results when more than a single mediator is involved. }\end{array}$ \\
\hline
\end{tabular}

than simply being a function of activating a single link between cue and target, recall is best described as the meshing of the associative networks activated by each of these entities (Nelson et al., 1998). In the extralist cuing task, recall appears to be the result of an intersection of the test cue and its activated associates with the target and its activated associates. However, when attempting to control or to manipulate a preexisting relationship between related words, investigators traditionally resort to free association norms and use only a single indicator for predicting connection strength. Typically, forward cueto-target strength is used as the only metric (e.g., Bahrick, 1970; Nelson \& McEvoy, 1979), but backward target-tocue strength has also been used (Humphreys \& Galbraith, 1975; Nelson et al., 1998) because it carries implications for the encoding specificity principle (Tulving \& Thomson, 1973). These metrics have proven their usefulness in predicting memory performance, but their unidimensional nature places limits on their effectiveness, given that we know that the connections relating any two words are likely to be much more complicated. An index that incorporates multiple sources of word-to-word connection strength will ease list construction and reduce confounding in any task in which word-to-word relationships are critical. Two summary indices were developed, and their effectiveness was evaluated in multiple regression analyses by determining how well they predicted the probability of correct extralist cued recall.

\section{METHOD}

\section{Procedure}

In what we will call the standard procedure for the extralist cuing task, a single study trial on a list of individually exposed target words was followed by a single self-paced test trial. During study, targets were presented visually at $3 \mathrm{sec}$ per word under intentional learning instructions indicating that memory for the words would be tested. No advance information about the nature of the test was provided. During the test trial, a single cue for each target was presented to prompt the recall of its target, and testing followed im- mediately after a short instructional period. All the subjects were given explicit test instructions indicating that they should use each test cue to help them recall one of the studied target words. Fiftyeight percent of the items were studied and tested under this standard condition; $23 \%$ were given intentional learning instructions, but the items were presented at faster or at slower rates; and 19\% were studied or tested under different instructions (concretenessrating and vowel-naming study instructions, or must-guess and must-not-guess test instructions).

\section{Materials}

The 2,272 cue-target pairs were taken from 29 different experiments run in our laboratory from 1989-1999. These pairs were selected from a large free association database that was started in the 1970 s that now includes free association responses to $5,000+$ words and their responses for a total of more than 72,000 pairs. These norms and the procedures for collecting them can be obtained on the Web (Nelson, McEvoy, \& Schreiber, 1999). Table 1 provides the definitions for the features of cues, targets, and cue-target pairs that were defined by using the norms and that were evaluated in these experiments, including set size, forward strength, and so forth. Table 2 presents the sources of the data, as well as an indication of what variables were manipulated in each experiment. The published studies are indicated by the last names of the authors, the dates of publication, and the specific experiments involved so that these references can be examined for additional details-for example, the specific pairs used in each experiment, the number of subjects, and so on. The unpublished studies are being prepared for publication and do not differ in procedures or in item selection procedures. Although target set size was manipulated more than any other variable, no single variable was manipulated in all the experiments. All the experiments had 24 pairs in each list, and at least two different lists were used in each one. An average of 10.61 subjects $(S D=3.19$, range $=8-24$ ) studied and were tested on each pair, with probability of correct recall computed by dividing the number of subjects who correctly recalled the target, relative to the total tested on that target.

Table 3 summarizes the experimental database in a way that closely parallels work with the individual experiments. The purpose of this table is to convey the main descriptive statistics for each feature analyzed in the multiple regression analyses to follow and to show that, when comparable criteria are used for defining features, they have similar effects in the database and the individual experiments. The first column indicates the selected features that were chosen because they had been manipulated in prior experiments and 
Table 2

Source of Materials

\begin{tabular}{ll}
\hline \multicolumn{1}{c}{ Source of Materials and Data } & \multicolumn{1}{c}{ What Feature Was Varied } \\
\hline Nelson, McEvoy, \& Schreiber (1990), Experiment 2 & TSS \\
Nelson \& Schreiber (1992), Experiments 1 \& 2 & TSS, TCON \\
Nelson, Schreiber, \& McEvoy (1992), Experiments 1 \& 3 & FS, QSS, TSS \\
Nelson, Schreiber, \& McEvoy (1992), Experiment 4 & QSS, TSS \\
Nelson, Bennett, Gee, Schreiber, \& McKinney (1993), Experiments 1, 1 A, 3-5 & TSS, TC \\
Nelson, Bennett, Gee, Schreiber, \& McKinney (1993), Experiment 2 & TSS, TC, SAS \\
Nelson, McEvoy, Janczura, \& Xu (1993), Experiments 1-3, 5 & TSS \\
Nelson \& Xu (1995), Experiments 1 \& 2 & TSS, TFREQ \\
Nelson, Bennett, \& Leibert (1997), Experiment 1 & FS,SAS-MS, TSS \\
Nelson, Bennett, \& Leibert (1997), Experiment 2 & MS-SAS \\
Nelson, McKinney, Gee, \& Janczura (1998), Experiment 1 & TC, TR \\
Nelson, McKinney, Gee, \& Janczura (1998), Experiment 2 & TSS, TC, SAS \\
Nelson, McKinney, Gee, \& Janczura (1998), Experiment 3 & FS, BS \\
Unpubiished & TSS, TR, TC \\
Unpublished & FS-BS-SAS-MS, TSS, TC \\
Unpublished & FS, TC \\
Unpublished & TC, TFREQ \\
Unpublished & TSS, Remote TSS \\
\hline
\end{tabular}

Note-OSS, cue set size; TSS, target set size; TC, target connectivity; TR, target resonance; FS, forward strength BS, backward strength; SAS, shared associate strength; MS, mediated strength; TCON, target concreteness; TFREQ, target frequency; Remote TSS, set size of the associates of the target.

had been shown to affect cued recall. The second column reports the average value of the specified feature for the 2,272 items used in the 29 experiments (actually, because of missing concreteness ratings, the sample sizes range from 2,171 to 2,272). Standard deviations are reported next to the means throughout the table. Hence, for cue set size, the data indicate that the 2,272 test cues had an average of 13.68 associates $(S D=5.20)$.

The next four columns report the standard criteria used for defining the variables in the individual experiments and the effects of these variables on the probability of correct cued recall. These criteria were always defined as less than, greater than, or equal to some fixed value determined by examining the frequency distribution for the feature and then selecting criteria at the extremes. For example, test cues and targets with small and large sets have been defined as those with 8 or fewer associates at the low end and those with 18 or more associates at the high end, respectively, as determined by free association norms. The third column reports the criteria used for each feature. The fourth column indicates the mean and standard deviation achieved for the feature at each criterion, and the fifth column indicates the item sample size that meets this criterion. Finally, the sixth column reports the probability of cued recali achieved at each criterion. To continue the cue set size example, cues with smaller sets tended to produce higher levels of recall (.63) than did those with larger sets (.49).

That all contrasts were significant was indicated by $t$ tests on the recall probabilities. Variables that affect cued recall in subjectbased analyses for data collected in individual experiments have the same effects in item analyses based on data pooled over subjects. Rather than describing each effect in detail, in order to conserve space, readers are advised to scan across the rows describing each feature to gain a sense of the nature of each manipulation, the number of items in the database that meet the criteria, and the effect of

Table 3

Features, Their Descriptive Characteristics, and Their Influence on Probability of Correct Recall

\begin{tabular}{|c|c|c|c|c|c|c|c|c|}
\hline \multirow[b]{2}{*}{ Feature } & \multicolumn{2}{|c|}{ Value } & \multirow[b]{2}{*}{ Criterion } & \multicolumn{2}{|c|}{ Manipulation } & \multirow[b]{2}{*}{ Sample Size } & \multicolumn{2}{|c|}{ Recall } \\
\hline & Average & $S D$ & & $M$ & $S D$ & & $P$ & $S D$ \\
\hline \multirow[t]{2}{*}{ Cue set size } & 13.68 & 5.20 & $\leq 8$ & 6.83 & 1.39 & 444 & .63 & .25 \\
\hline & & & $\geq 18$ & 20.97 & 2.43 & 526 & .49 & .25 \\
\hline \multirow[t]{2}{*}{ Target set size } & 13.80 & 7.12 & $\leq 8$ & 6.60 & 1.42 & 990 & .61 & .25 \\
\hline & & & $\geq 18$ & 21.37 & 2.44 & 937 & .47 & .26 \\
\hline \multirow{2}{*}{ Target frequency } & 99 & 177 & $\leq 15$ & 7 & 5 & 694 & .60 & .25 \\
\hline & & & $\geq 100$ & 288 & 260 & 601 & .50 & .26 \\
\hline \multirow[t]{2}{*}{ Target concreteness } & 4.90 & 1.29 & $\leq 3$ & 2.61 & 0.37 & 243 & .47 & .25 \\
\hline & & & $\geq 5$ & 5.89 & 0.45 & 1,243 & .57 & .26 \\
\hline \multirow[t]{2}{*}{ Target connectivity } & 1.54 & .79 & $\leq .85$ & .56 & .20 & 534 & .53 & .27 \\
\hline & & & $\geq 2.20$ & 2.68 & .38 & 473 & .60 & .24 \\
\hline \multirow[t]{2}{*}{ Target resonance } & .53 & .24 & $\leq .36$ & .23 & .08 & 580 & .48 & .27 \\
\hline & & & $\geq .66$ & .82 & .11 & 649 & .60 & .25 \\
\hline \multirow[t]{2}{*}{ Forward strength } & .17 & .12 & $\leq .08$ & .06 & .02 & 880 & .46 & .26 \\
\hline & & & $\geq .24$ & .35 & .09 & 505 & .69 & .22 \\
\hline \multirow[t]{2}{*}{ Backward strength } & .12 & .20 & $\leq .08$ & .02 & .02 & 1,465 & .50 & .26 \\
\hline & & & $\geq .24$ & .51 & .20 & 361 & .65 & .23 \\
\hline \multirow[t]{2}{*}{ Shared associate strength } & .04 & .08 & $\leq .005$ & .001 & .002 & 897 & .48 & .27 \\
\hline & & & $\geq .05$ & .15 & .10 & $48 !$ & .65 & .25 \\
\hline \multirow[t]{2}{*}{ Mediated strength } & .03 & .05 & $\leq .005$ & $.00 !$ & .001 & 1,018 & .50 & .27 \\
\hline & & & $\geq .05$ & .12 & .07 & 372 & .67 & .23 \\
\hline
\end{tabular}


Table 4

Correlations Among the Attributes of Associative Networks

in the Normative Database and in the Experimental Database

\begin{tabular}{lccccccccrr}
\hline & \multicolumn{10}{c}{ Attributes } \\
\cline { 2 - 10 } & QSS & TSS & TFREQ & TCON & TC & \multicolumn{1}{c}{ TR } & FS & BS & SAS & MS \\
\hline QSS & - & .06 & .02 & -.09 & .01 & -.07 & -.26 & -.08 & -.23 & -.20 \\
TSS & .13 & - & .06 & .10 & .25 & -.21 & .00 & -.13 & -.20 & -.07 \\
TFREQ & -.02 & .25 & - & -.23 & -.14 & .39 & .01 & -.19 & -.17 & .09 \\
TCON & -.07 & -.06 & -.28 & - & .02 & .08 & .07 & .02 & .04 & .03 \\
TC & .05 & .32 & .01 & -.01 & - & .01 & -.01 & -.01 & .07 & .00 \\
TR & -.13 & -.26 & .29 & .12 & .01 & - & .11 & -.12 & -.06 & .24 \\
FS & -.23 & .01 & .17 & .03 & -.03 & .29 & - & .29 & .03 & .17 \\
BS & .04 & -.38 & -.30 & .04 & -.12 & -.08 & -.01 & - & .04 & -.05 \\
SAS & -.30 & -.33 & -.16 & .02 & -.04 & .04 & -.08 & -.09 & - & .40 \\
MS & -.23 & -.16 & .16 & .05 & -.06 & .34 & .17 & -.19 & .43 & - \\
\hline
\end{tabular}

Note-Values above and below the diagonal are from the normative and the experimental databases, respectively. QSS, cue set size; TSS, target set size; TFREQ, [ $\log (.5+$ target frequency)]; TCON, target concreteness; TC, target connectivity; TR, target resonance; FS, forward strength; BS, backward strength; SAS, shared associate strength; MS, mediated strength.

the feature on recall. We draw attention to two separate trends. First, as indexed by concreteness ratings for test cues and targets, the word sample is biased toward concrete words, as opposed to more abstract ones. Concreteness was investigated early on in the project, and as a result of this work, this variable was routinely computed and was not allowed to be confounded with any of the variables that were being manipulated. Second, although the same criteria were used to define low and high levels of forward and backward strength, the latter varied over a wider range, particularly because high back strength items tended to have stronger connections than did high forward strength items. Back strength, as will be discussed below, was not investigated until later in the project, and unfortunately, it was not always carefully controlled, despite evidence (Humphreys \& Galbraith, 1975) and theory (Tulving \& Thomson, 1973 ) indicating that it could be important.

\section{RESULTS}

\section{Correlations Among the Features}

Table 4 presents the correlations between the features in the normative database (above the diagonal) and those in the experimental database (below the diagonal) that will be evaluated in the main regression analysis. Except for concreteness, which was based on a sample of 51,753 , the normative values shown in Table 4 were based on 58,143 pairs of words consisting of the 5,019 normed words (cues) and their responses (targets). For this analysis, only targets having their critical associates normed were included, so this sample represents $81 \%$ of the pairs in the free association norms (Nelson, McEvoy, \& Schreiber, 1999).

Generally speaking, the correlations among the features in the normative database were low, suggesting that they were largely independent, but because of the large sample size, any correlation greater than .01 was significant after Fisher's $r$-to- $z$ transformation. Despite the low correlations, several patterns emerged, and they were similar for cues and for targets normed independently as cues. For example, test cues with larger associative sets tended to be more weakly related to their responses than were those with smaller sets. The correlations of cue set size with forward, backward, shared associate, and mediated strength were small and negative. Similarly, with the exception of forward strength, the correlations of target set size with strength measures tended to be small and negative.

Although words with smaller sets tend to have more connections from their associates back to the target (resonance) and fewer connections among their associates (connectivity), the correlations are relatively small ( $r=$ -.21 and .25 , respectively). Table 4 also shows that weak natural relationships emerged among some of the indices of strength. Positive correlations were obtained for forward-backward strength $(r=.29)$, for forwardmediated strength $(r=.17)$, and for mediated-shared associate strength $(r=.40)$. The magnitude of the forwardbackward relationship indicates that, although associative symmetry is relatively common in natural language, asymmetry is the more typical pattern when strength is indexed through free association. In addition, the correlation among the two indirect indices of strength suggests that many pairs are related both because the two words produce the same associate and also because they share a two-step mediator.

The lower half of Table 4 presents the correlations among the attributes for the pairs used in various experiments for which all 10 indices were available. In general, these values appear to be relatively low and of magnitudes approximately similar to those in the normative database. The correlation between the 45 correlations associated with the two databases was $r=.84$, which was significant $(z=7.94)$. Ideally, if perfect control had been attained in the experimental database, the 45 correlations among the attributes would have been zero in every case, and the overall correlation between the two databases would have been lower. This was unfortunately not the case, and several troublesome discrepancies emerged. The one raising the most problems indicated that target set size had been unintentionally and partially confounded with backward target-to-cue strength and, to a lesser extent, with shared associate and mediated strength. Targets with smaller sets tended to be more strongly connected to their test cues. In addition, their resonance tended to be higher, and the 
Table 5

Results of the Multiple Regression Analysis Evaluating Probability of Correct Cued Recall as a Function of Each Attribute

\begin{tabular}{lccr}
\hline \multicolumn{1}{c}{ Attribute } & Standard Error & Standard Coefficient & $t$ Value \\
\hline Intercept & .035 & .313 & 8.86 \\
Cue set size & .001 & -.039 & -2.00 \\
Target frequency & .008 & -.110 & -6.22 \\
Target concreteness & .004 & .086 & 4.49 \\
Target set size & .001 & -.151 & -4.97 \\
Target connectivity & .006 & .189 & 9.92 \\
Target resonance & .025 & .031 & 1.38 \\
Forward strength & .041 & .370 & 18.99 \\
Backward strength & .028 & .206 & 9.82 \\
Shared associate strength & .076 & .081 & 3.59 \\
Mediated strength & .106 & .147 & 6.77 \\
\hline
\end{tabular}

connectivity of their associates tended to be slightly lower. These partial confoundings emerged because the earlier experiments ignored backward strength and indirect strength, as well as connectivity and resonance strength, in constructing the lists. By way of contrast, the effects of forward strength were known early on in the research (e.g., Bahrick, 1970; Nelson \& McEvoy, 1979), and this factor was always controlled or manipulated so it was never confounded with target set size. Eventually, the influence of the other features in the associative cuing task were recognized or discovered, and as they were, tighter control was achieved. In what follows, we present the results of multiple regression analyses in an effort to disentangle the separate effects of these variables.

\section{Main Regression Analysis}

Table 5 presents the results of the multiple regression analysis of the 10 attributes used for predicting probability of correct cued recall. The assumptions underlying this regression were evaluated by examining the residuals (Myers \& Well, 1995, pp. 406-410). Residual probabilities of correct recall were plotted against fitted probabilities of recall for each attribute, and each plot showed the residuals to be evenly and linearly related to fitted probability. Frequency distributions of the residuals closely approximated normal distributions, and the results of the Durbin-Watson $(D)$ statistic, as well as the serial autocorrelations, provided no evidence for lack of independence. The $D$ statistics for the 10 attributes averaged 1.49 , with the largest deviation from this value being 1.52 , and the serial autocorrelations averaged .26 , with the largest deviation being . 24 . Hence, the analysis of the residuals suggested that assumptions of linearity, homogeniety of variance, normality, and independence were reasonable for each attribute entered into the equation.

The results of the main multiple regression analysis indicated that the 10 features were significantly related to recall $\left[R=.58 ; F(10,2120)=107.23, M S_{\text {res }}=.045\right]$. Table 5 presents the standard errors, the standardized coefficients, and the $t$ values for the intercept and each feature. The standardized regression coefficients shown in the middle column of Table 5 are calculated as if all of the independent variables had means of zero and standard deviations of one. Such coefficients can be compared re- gardless of the differences in the scales of the variables involved, and they can be used to determine which attributes in the regression equation contributed most to predicting cued recall. In the present database, forward strength (.37) contributed the most, followed by backward strength $(.21)$ and target connectivity (.19) and then by target set size $(-.15)$ and mediated strength (.15). Frequency $(-.11)$, shared associate strength $(.08)$, and concreteness $(.09)$ contributed about equally to recall, and cue set size $(-.04)$ also produced a small but significant effect. Target resonance (.03) had a small positive effect that was not reliable.

Other than reducing the overall correlation $(R=.56)$ and reducing the differences between the two measures of indirect strength, deleting concreteness and frequency had little influence on the results of the main regression. The main results were also unaffected by restricting the analysis to nouns ( 1,285 cue-target pairs), nor were they influenced in any significant way when additional cue characteristics were added to the 10 characteristics used in the main analysis (i.e., cue frequency, resonance, and connectivity). Neither cue frequency nor cue resonance had significant effects. Although cue connectivity had a significant positive effect, this variable has never been investigated in a manipulational design, so the specific findings were omitted in order to conserve space.

Furthermore, deleting data obtained under vowelnaming instructions had little effect on the main regression analysis, but we note that these instructions produced interesting contrasts in effect sizes when separate regression analyses were conducted for each instructional condition. Concreteness rating instructions ( $n=186$ targets) produced results that were nearly identical to those of standard intentional learning instructions $(n=1,872$ targets). In contrast to both of these instructional sets, vowelnaming instructions ( $n=138$ targets) produced smaller beta coefficients for target set size, connectivity, backward strength, and shared associate strength and larger betas for forward strength and mediated strength. In short, the poor encoding associated with vowel naming appeared to decrease reliance on information encoded about the target and to increase reliance on information provided directly by the test cue. Finally, we note that an unforced stepwise regression ( $F$-to-enter $=2.41$ and $F$-toremove $=2.39$ ) of the same data produced results that 
were similar to those of the multiple regression analysis in that only resonance was left out of the model. Each feature added significantly to explained variance after prior features were accounted for. They emerged in steps in the following order: forward strength, target set size, target connectivity, backward strength, target frequency, mediated strength, target concreteness, shared associate strength, and cue set size. Thus, target set size contributed significantly to recall probability after the effects of forward strength were accounted for, and so forth.

The regression results confirmed what previous individual experiments had shown when these variables were manipulated in the extralist cued recall task (e.g., Nelson \& McEvoy, 2000; Nelson \& Schreiber, 1992; Nelson \& $\mathrm{Xu}, 1995$ ). More important, they show that each feature affects recall in the expected direction when the influence of the remaining features has been controlled statistically by multiple regression. Cue and target set size have significant negative effects on cued recall (Nelson, Schreiber, \& McEvoy, 1992). Targets are more likely to be recalled when they are concrete (Nelson \& Schreiber, 1992) and when they occur less frequently (Nelson \& McEvoy, 2000; Nelson \& Xu, 1995). The results also indicate that some features contribute more to correct recall than do others. Direct forward connections between the test cue and the target have the largest effect on cued recall, followed by backward connections and then by indirect connections (Nelson, Bennett, \& Leibert, 1997). In addition, connections among the associates of the target (Nelson, Bennett, Gee, Schreiber, \& McKinney, 1993) contribute more than resonant connections (Nelson et al., 1998) and have about the same influence as target set size effects (Nelson, Schreiber, \& McEvoy, 1992).

\section{PROPORTION OF VARIANCE EXPLAINED}

The purpose of this section is to determine how much of the variance in extralist cued recall can be explained by indices of word knowledge taken from free association. The results of a regression analysis that included eight free association indices indicated that a linear combination of these measures was correlated .56 with probability of cued recall, producing an adjusted $R^{2}$ of .32 (target concreteness and frequency were omitted in this analysis). Thirty-two percent of the total variance in the extralist cued recall task can be predicted from free association measures taken before an experiment. This estimate, however, is also affected by the variance associated with each task, so it is specific to this data set (Myers \& Well, 1995, pp. 394-395, 472-473). A more accurate estimate takes the reliability of each task into account, which then sets the ceiling for the correlation. The reliability of each task was assessed by using test-retest procedures.

In the discrete free association task, two separate reliability studies have been conducted by renorming the same words on different samples of subjects. Nelson and Schreiber (1992) renormed 155 items, and Nelson et al. (2000) renormed 120; each investigation produced the same average reliability of 89 . To estimate the reliability of the extralist cued recall task, identical cue-target pairs learned under similar encoding conditions in different experiments were culled from the present study. The probability of correct cued recall was calculated for the first and second occurrences of the pair, with individual pairs randomly assigned to first and second positions having averages of 10.91 and 11.07 subjects per pair. This procedure amassed $225 / 2,272$ pairs and was possible because some of the pairs were used in more than one experiment. Small differences in encoding conditions were allowedfor example, the same words presented at 3- or 4-sec presentation rates could be paired, as could concretenessrating and intentional learning instructions, and in most cases, the same pair was drawn from a different list context. If anything, allowing slight differences was expected to make the estimate of reliability more conservative, and given these constraints, reliability was estimated to be .73 . Given the observed correlation between free association and cued recall of .56 in the multiple regression analysis and respective estimates of reliability of .89 and .73 , the true correlation between free association and extralist cued recall is $.70 .{ }^{1}$ Measures taken from free association predict approximately $49 \%$ of the variance of extralist cued recall when the reliabilities of the two tasks have been taken into account. Hence, nearly one half of the variance in cued recall can be attributed to prior knowledge, as indexed by free association.

\section{MEASUREMENT ALGORITHMS}

The results of the main regression analysis indicated that, although they produced different effect sizes, 10 variables related to preexisting lexical features influenced the likelihood of extralist cued recall. Knowledge of this information will be useful to anyone working on cuing or priming tasks involving related words, but such knowledge also means that manipulating one of these variables requires controlling the others. Given the availability of a large normative database (Nelson, McEvoy, \& Schreiber, 1999), this task is as possible as it is dreadful to implement. A means for reducing some of these variables to single predictors would be useful, and it also would be helpful to have key indices expressed in the same form. For example, in the individual experiments, the indices of resonance and connectivity were based on counting links among the items making up the targets' associative sets. Counting the links proved to be an efficient way to capture these variables, but such counts effectively assign a connection strength of unity to each link, when in fact the links vary in strength, just as with any word-to-word association. Expressing these variables in terms of a strength index would take such variation into account and would put the indices of resonance and connectivity more on a par with indices of direct and indirect strength. However, unlike single-connection measures such as forward strength, resonance and connectivity are likely to involve many connections, and some theoretically driven means of combining them into a single index 
is required. In this paper, we used an equation associated with PIER 2 to begin this process. ${ }^{2}$

PIER 2 purports to explain how preexisting knowledge about word relationships affects cued recall and offers a principled means for data reduction (Nelson et al., 1998). Generally speaking, the process aspects of the model attribute cued recall to the computation of the intersection of the test cue and its associates with the target and its associates. The formal aspects of the model rely on a set of three equations that estimate net cue-target strength. The first equation was designed to capture the influence of resonance and connectivity, the second incorporates the effects of direct and indirect connection strengths, and the third addresses the negative effects that set size has on cued recall. These equations constitute a theory about implicitly activated associates and their role in incrementing the activation level of a studied word in long-term working memory (LTWM). With the addition of other processing assumptions and parameters, these equations can be implemented as a process model that is evaluated through simulation procedures (e.g., Raaijmakers \& Shiffrin, 1981). In addition and more germane to the present aims, these equations can be implemented as measurement algorithms designed to reduce multiple sources of strength to single values that can be used to predict performance in cued recall and other tasks. Such an implementation represents a next step in the tradition of controlling or manipulating forward and backward strength in order to predict cued recall (Bahrick, 1970; Nelson et al., 1998; Tulving \& Thomson, 1973) and priming (e.g., Kahan, Neely, \& Forsythe, 1999). It is a next step because, instead of using only a single source of strength, the model can be used to incorporate the combined effects of two or more variables in a single index. In this paper, we focus on Equation 1 and only briefly describe the results of incorporating the results of this equation into Equation 2.

\section{Equation 1}

The rationale underlying Equation 1 assumes that comprehension processes associated with reading the target during study automatically activate an implicit representation of the target and its associates in LTWM. The strength of this representation increases with activation returning to the target from its associates (resonance), as augmented by connections among the associates (connectivity). The equation can be most easily understood as attributing resonance and connectivity effects to activation that spreads back to the target from its associates (J. R. Anderson \& Pirolli, 1984). More formally and in its most elementary form, the strength of the target as an implicit representation, $S\left(T_{i}\right)$, is

$$
S\left(T_{i}\right)=a_{i}+\sum_{j}^{n} a_{j} r_{i j}
$$

where $a_{i}$ is the activation of the implicit representation of the target $i$ as a result of its presentation, and $a_{j}$ is the ac- tivation of associate $j$ occurring as a result of presenting the target. Furthermore,

$$
r_{i j}=w_{i j}+\sum_{k}^{n} a_{k} r_{j k}
$$

and it represents the resonance coming back to target $i$ from associate $j$ as modified by associate-to-associate connections within the set for all $w_{i j}>0$, and for $j \neq k$, $k \neq i$. $w_{i j}$ is the preexisting link coming back to target $i$ from associate $j$. Note that the $w_{i j}>0$ restriction indicates that the influence of connectivity on a given associate is zero unless that associate is linked back to the target.

Equation 1 can be implemented as a measurement algorithm because it depends on word-to-word connections among the target's associates, and the mean preexisting strength of each of these links can be estimated by using free association norms (see Nelson et al., 2000, for the theory underlying these estimates). A complete example calculation of this use of Equation 1 is provided in Appendix A. The first task in evaluating the usefulness of such calculations was to determine how strongly they were related to the connection count indices that they would be replacing. For example, if Equation 1 effectively captures the indices of resonance and connectivity, it should be positively correlated with each index. Unfortunately, this was not the case. Although it was positively correlated with resonance $(r=.62, n=2,131)$, it was negatively related to connectivity $(r=-.21)$. Words with more connections among their associates tended to have weaker resonant connections $(r=-.19)$. What is more, dropping the $w_{i j}>0$ restriction had virtually no effect on the outcome. Hence, even when the resonance connection assumption has been relaxed, Equation 1 predicts that connectivity will have a negative effect on recall, a prediction that stands in contrast to the results of the main regression analysis.

\section{Equation 1A}

Given the goal of developing a strength index that effectively incorporated both resonance and connectivity, we sought an alternative formulation that could serve the same purpose as Equation 1 in PIER 2 but that was more strongly related to connectivity. In one variation that was far more simple to calculate than Equation 1, the computation of preexisting target strength was implemented by adding its nominal strength to the strengths of its associates and its resonant and associate-to-associate connections. More formally, the strength of the target is indexed as

$$
\begin{aligned}
S\left(T_{i}\right)= & {\left[S(T, T)+\sum_{i=1}^{n} S\left(A_{i}, T\right)\right] } \\
& +\sum_{j=1}^{n}\left[S\left(T, A_{j}\right)+\sum_{i=1}^{n} S\left(A_{i}, A_{j}\right)\right],
\end{aligned}
$$

where $S(T, T)$ represents the strength of the target's representation as a result of its presentation (nominally set 
to 1.00$), S\left(A_{i}, T\right)$ is associate-to-target or resonance strength, $S\left(T, A_{j}\right)$ is the strength of the target-to-associate links, and $S\left(A_{i}, A_{j}\right)$ is the strength collected from associateto-associate connections, or what we call connectivity. In the second part of the equation, the summed strength acquired by each associate in the set is determined by the sum of the inputs from the target and from other associates in the set. A computational example is shown in Appen$\operatorname{dix} \mathrm{B}$, using estimates of connection strengths based on the free association norms, as was done for Equation 1 in Appendix A. What is interesting about Equation $1 \mathrm{~A}$ is that connectivity among the associates adds directly to target strength and exerts an influence independent of resonance.

Equation 1A was positively correlated $(n=2,131)$ with the connection count indices it was meant to replace (resonance, $r=.21$, and connectivity, $r=.58$ ). It was also more strongly related to connectivity than to resonance, a result that was in accord with the main regression analysis. These correlations indicated that Equation $1 \mathrm{~A}$ captured the indices of resonance and connectivity better than did Equation 1. When Equation $1 \mathrm{~A}$ was substituted for the two connection count indices in the main regression analysis while leaving all of the other features in the regression (e.g., frequency, set size, and so on), the overall predictability was little changed $(R=.56 ; F=108.40$, $\left.M S_{\mathrm{c}}=.05\right)$. The beta coefficient for Equation $1 \mathrm{~A}$ was .16 which was significantly related to cued recall, and the beta coefficients for the other features were not appreciably altered; as compared with the main regression analysis. Finally, adding the square of this equation to this regression analysis increased $R$ by only .006 , which suggests that the relationship between Equation $1 \mathrm{~A}$ and probability of recall is adequately represented by a linear function.

\section{Equation 2}

The results of Equation $1 \mathrm{~A}$ were folded into the computation of PIER 2's Equation 2 for each word pairing in the experimental database. Equation 2 computes net cue-target strength and is defined as

$$
S\left(Q_{j}, T_{i}\right)=\sum_{k}^{n} S_{j k} S_{i k}+\sum_{k}^{n} S_{j k} S_{k i},
$$

where $Q_{j}$ is the test cue, $T_{i}$ is the target, and $k$ are the associates that join the cue and the target through convergent and mediated links (Nelson et al., 1998). When implemented as a measurement algorithm, Equation 2 computes the intersection between the test cue and its associates and the target and its associates by cross-multiplying word-toword connection strengths involving the target, the test cue, and the associates that indirectly link them together. An example calculation is shown in the lower part of Appendix $B$. The results were then entered into a regression analysis in place of the indices for target resonance and connectivity and the four separate indices of cue-target strength. Set size, concreteness, and frequency were included, as in the main analysis. This regression was significant $\left[R=.55 ; F(5,2125)=183.76, M S_{\text {res }}=.05\right]$, with Equation 2 accounting for the majority of the variance (standardized beta $=.46, S E=.006$ ). The standardized betas were similar in magnitude to those obtained in the main regression analysis of the 10 variables and were the following: cue set size, $-.02(S E=.001)$, target set size, $-.27(S E=.001)$, concreteness, $.09(S E=.004)$, and frequency, $-.09(S E=.007)$. Each beta was significant, except for cue set size.

Adding the square of Equation 2 to this regression significantly increased $R$ to .54 . Although this .02 increase in $R$ was statistically significant, it was numerically small. suggesting that the linearity assumption remains reasonable for the ranges of Equation 2 found in the present database. Nevertheless, a caution is in order. Forward cue-totarget strength was the best predictor of recall for this database, but $80 \%$ of the pairs had strengths of less than .26. Higher values were purposely avoided in order to avoid ceiling effects on probability of recall; had we allowed the full range of strength for each type of connection, we have no doubt that Equation 2 would have shown a stronger curvilinear relationship with recall. Under such conditions, adding two or more high-value connection strengths would no longer be equivalent to adding two or more low-value connection strengths, because of differences in scale. Under such conditions, Equation 2 would begin to fail as a predictor. Hence, Equation 2 represents an effective summary index of six separate features, because it successfully explains a substantial portion of the variance of extralist cued recall, but its usefulness as a predictor is limited to relatively weaker pairings. Only under such conditions is the linearity assumption likely to hold.

\section{DISCUSSION}

The extralist cuing task involves a mix of episodic learning and cuing based on preexisting information. Unlike paired-associate tasks, the test cues are unavailable during the study episode, and subjects must rely on what they know about the relationship between cues and their targets to achieve specified retrieval goals. In many ways, the task mimics everyday cue-dependent memory performance and provides an ideal method for studying the intersection between known and new information. However, although the conditions under which the new information is acquired can be manipulated and otherwise carefully controlled, what is supposedly known about the cue-target relationship can only be inferred from measurements taken from other sources. Examples of these sources include concreteness ratings (Paivio, Yuille, \& Madigan, 1968), frequency counts (Kučera \& Francis, 1967), and free association (Bahrick, 1970; Nelson et al., 1998). In these and other examples, measurements are taken from some individuals in order to infer what other individuals are likely to know.

The success of this enterprise is normally determined by whether a particular feature identified through the measurement procedure has an effect on task performance. In the extralist cuing task, many features of lexical knowledge have been identified and measured through the use of free association and have been shown 
to affect recall in this task (e.g., Nelson et al., 1998; Nelson, Schreiber, \& McEvoy, 1992). In this sense, this approach to the investigation of the intersection of known and new information has been successful. Nevertheless, a higher criterion for success requires that the individual features so identified exert their influence above and beyond their relationships with other features (Rubin \& Friendly, 1986). For example, the effect of the size of a word's associative set on recall has been known for many years (e.g., Nelson \& McEvoy, 1979). Extensive empirical work has shown that this effect is independent of concreteness (Nelson \& Schreiber, 1992), frequency (Nelson \& Xu, 1995), connectivity (Nelson et al., 1993), and word ambiguity (Gee, 1997). Despite all of this work, set size has not been investigated with respect to other word features (e.g., backward strength), but what is more important, it has not been investigated in the context of simultaneous variations in all of the lexical features known to affect cued recall.

An experiment that crossed 10 or more features simultaneously would be as impractical to do as it would be impossible to understand. There are clear limits to individual experiments when it comes to investigating the simultaneous effects of a constellation of variables known to affect performance. In the present article, this dilemma was circumvented through the use of correlational and multiple regression analyses on a database comprising thousands of cue-target pairings taken from a large number of individual experiments. The correlational analyses for the 10 features representing the main focus of the article indicated that, for the most part, the correlations among the features were low and reflected the magnitude of the relationships observed in the much larger normative database. Generally speaking, the results of correlational analyses using the normative database suggest that the 10 features are mostly independent. In the experimental database, forward strength was never confounded with target set size as a result of direct attempts to control this relationship. Mostly by accident, forward strength was never confounded with back strength, even though a modest correlation exists in the normative database $(r=.29)$. The one important exception to this pattern of low correlations involved target set size and back strength. These variables are weakly correlated in the normative database $(r=-.15)$ but more strongly correlated in the experimental database $(r=-.39)$, because back strength was left uncontrolled in most of these experiments. Target set size and back strength were partially confounded in the database, and although hardly a new idea, it is useful to be reminded, at least occasionally, that manipulating one feature while letting another vary randomly provides no guarantee against confounding them, even when they are poorly related in natural language.

Despite the partial confounding of target set size and back strength and other weak relationships, the results of the multiple regression analyses indicate that each of the features manipulated in these experiments contribute to the probability of extralist cued recall. Multiple regression adjusts for correlations among variables, so it is possible to estimate the relative contributions of each feature to recall by using standard beta coefficients. These coefficients indicated that forward strength has the largest effect on extralist cued recall, followed by backward strength, connectivity, set size, indirect measures of strength, concreteness and frequency, and lastly, by target resonance. As was expected, each feature has positive effects on recall, except cue and target set size and target frequency, which have negative effects on recall. These results were largely unaffected by various restrictions placed on the database, such as limiting the items to nouns. What is most important, these results confirm what individual experiments have shown. Features that affect recall in individual experiments manipulating two or three features at a time have the same effects on recall in multiple regression involving simultaneous variation in all of the features. The findings are robust in that they generalize over different subjects with similar cultural backgrounds and over different items with similar associative characteristics. Finally, when task reliabilities are taken into account, $49 \%$ of the variance in extralist cued recall appears to be determined by preexisting knowledge about the test cue and target and about the associative links that connect them.

Both the individual experiments and the multiple regression analyses identify a collection of word features that influence extralist cued recall, and it would be practically and theoretically useful to capture this influence in an algorithm that combines at least some of these features into single predictors. Early theoretical work with this task suggested that both forward cue-to-target strength and backward target-to-cue strength would be important. The present findings recapitulate a portion of the developmental history of research on extralist cued recall, in which proponents of generation-recognition models stressed the importance of forward strength (Bahrick, 1970), whereas proponents of encoding specificity stressed the importance of back strength (Tulving \& Thomson, 1973). The encoding specificity principle stated that the test cue had to be encoded while the target was being encoded in order for it to be a successful cue. Each group was correct, as the present findings indicate that these features represent the best overall individual predictors of recall success. Of course, neither approach can explain as much variance as PIER 2's Equation 2 implemented as a measurement tool, because it incorporates six different sources of strength. Two of these sources, resonance and connectivity, concern target activation strength, and four of these sources concern direct and indirect connections linking the cue and the target. This implementation depends on using free association norms to estimate word-toword connection strengths and on the theory underlying the model for determining how to combine these strengths into a single index.

PIER 2's Equation 1 was used as a means for expressing the connection count indices for resonance and connectivity as an index of target strength. However, this equation failed to capture connectivity in a way that reflected the influence of this variable on recall. The equation assumes 
that the activation level of the target increases with the strength of the resonant connections coming to it from its associates, as augmented by connectivity among the associates themselves. Connectivity comes into play indirectly by boosting resonance returning to the target. The feasibility of Equation 1 as a measurement index was evaluated by calculating it for each target in the database, using indices of word-to-word connection strengths provided by free association. Our expectation was that this equation would have to be positively correlated with connection count indices of resonance and connectivity in order for it to be useful for predicting the effects of these variables on recall. However, although Equation 1 was positively correlated with resonance, it was negatively correlated with connectivity and predicted that this variable would have a negative rather than a positive effect on recall.

In light of this problem, Equation 1 was redesigned to avoid the dependent relationship between connectivity and resonance. In Equation 1A, the sum of the target-toassociate strengths was added to the sum of the resonant strengths and the sum of the strengths stemming from associate-to-associate links. The result was used as an index of the activation strength of the target and its associates. Theoretically, this equation can be conceptualized as a parallel activation process, with all the connections in an associative network contributing additively to strengthening the target and its associates. In comparing the two equations, one key difference is that Equation $1 \mathrm{~A}$ allows associate-to-associate connectivity to contribute directly to target activation strength, whereas Equation 1 permits only an indirect contribution through resonant connections. This difference turned out to be critical, because Equation 1A was positively correlated with resonance and with connectivity and was more strongly related to connectivity. When this equation was substituted for the separate connection count indices of resonance and connectivity in the regression analysis, the results indicated that it significantly predicted probability of recall.

When the results of Equation 1A were fed into PIER 2's Equation 2, a single predictor was derived from six separate indices involving pairwise connections (resonance, connectivity, forward strength, backward strength, mediated strength, and shared associate strength). This new index of cue-target strength predicted probability of cued recall about as effectively as when the indices were separately entered into the regression equation. At least for the extralist cued recall task, a single value produced by a theoretically based algorithm can take the place of six separate measures. In selecting word pairs for experiments, this rule is likely to ease list construction, as well as to reduce confounding.

However, two caveats apply to using this equation for predicting word relatedness. First, its use should be confined to weak cue-target pairs in order to maintain linearity of scale. Second, the rule may or may not work for other tasks involving pairs of related words. The value of the equation has been derived solely from data on extralist cued recall, and its generalization can be determined only when we know whether performance in other tasks depends on computing the same sources of connection strength. For instance, this implementation of Equation 2 should not effectively predict target recovery under implicit conditions of learning and testing. Under implicit conditions, subjects are encouraged to produce the first word that comes to mind that is related to the test cue, and it is unlikely that they will be searching for a specific word recently seen in the testing context. Under this relaxed retrieval criterion, target recovery is more prone to be the result of priming the target as an associate of the test cue, as opposed to computing a cue-target intersection, and therefore, it is more likely to be influenced by forward and mediated links than by backward and shared associate links. Similarly, it is clear that lexical decision is a function of the forward and backward strengths of the prime-target pairs (e.g., Kahan et al., 1999), but we do not know whether indirect connections and connectivity will affect decision time, nor whether forward and backward connections will have additive effects. In these cases, a less complex rule or the indices of the individual features may function better than an algorithm that includes all the sources of connection strength. For the present, one main advantage of Equation 2 is that it alerts researchers to the different types of connections that link words together, while providing a structured algorithm for exploring their influence in a variety of different cognitive tasks involving related words.

By way of final comment, we note that implementing PIER 2 as a measurement model follows in a long tradition of using free association data to estimate the strength of preexisting connections among word pairs in order to forecast the effectiveness of various types of priming cues. PIER 2 implemented as a measurement tool follows in this tradition and is different only in that it has been designed to express multiple sources of connection strength as a single value, as opposed to using only a single source of strength while ignoring others. One difficulty inherent in this approach is that there is more than one way to incorporate the influence of multiple sources of information, and as the contrast between Equations 1 and $1 \mathrm{~A}$ makes clear, theories are required in order to make reasoned decisions about how to combine these sources. The present findings indicate that an equation that indexes connectivity independent of resonance will be more likely to capture the effects of this word feature than will one that assumes that it must be expressed through resonant connections. This conclusion, in turn, indicates that implementing PIER 2 as a measurement algorithm carries implications for developing it further as a process model designed to explain the effects of the same variables. We are now more wary of the assumption that activation must spread back to the target in order to heighten its activation and lean more toward one that allows connectivity among the target's associates to contribute independently. At the least, the present results suggest that each conceptualization of target activation strength needs to be modeled and evaluated on its merits. 


\section{REFERENCES}

Anderson, J. R., \& Pirolli, P. L. (1984). Spread of activation. Journal of Experimental Psychology: Learning, Memory, \& Cognition, 10 $791-798$.

Anderson, M. C., \& Spellman, B. A. (1995). On the status of inhibitory mechanisms in cognition: Memory retrieval as a model case. Psychological Review, 102, 68-100

Bahrick, H. P. (1970). Two-phase model for prompted recall. Psychological Review, 77, 215-222.

DEESE, J. (1965). The structure of associations in language and thought. Baltimore: Johns Hopkins University Press.

GEE, N. R. (1997). Implicit memory and word ambiguity. Journal of Memory \& Language, 36, 253-275.

HumphrEYS, M. S., \& GALBRAITH, R. C. (1975). Forward and backward associations in cued recall: Predictions from the encoding specificity principle. Journal of Experimental Psychology: Human Learning \& Memory, 1, 702-710.

Jenkins, J. J., \& RuSSELl, W. A. (1952). Associative clustering in recall. Journal of Abnormal \& Social Psychology, 47, 818-821.

Kahan, T. A., Neely, J. H., \& Forsythe, W. J. (1999). Dissociated backward priming effects in lexical decision and pronunciation tasks. Psychonomic Bulletin \& Review, 6, 105-110.

KuČERA, H., \& Francis, W. N. (1967). Computational analysis of present-day American English. Providence, RI: Brown University Press.

McEvoy, C. L., Nelson, D. L., \& Komatsu, T. (1999). What is the connection between true and false memories? The differential roles of interitem associations in recall and recognition. Journal of Experimental Psychology: Learning, Memory, \& Cognition, 25, 1177-1194.

Myers, J. L., \& WelL, A. D. (1995). Research design and statistical analvsis. Hillsdale, NJ: Erlbaum.

Nelson, D. L., Bennett, D. J., Gee, N. R., Schreiber, T. A., \& MCKINNEY, V. (1993). Implicit memory: Effects of network size and interconnectivity on cued recall. Journal of Experimental Psychology: Learning, Memory, \& Cognition, 19, 747-764.

Nelson, D. L., Bennett, D. J., \& Leibert, T. W. (1997). One step is not enough: Making better use of association norms to predict cued recall. Memory \& Cognition, 25, 785-796.

Nelson, D. L., \& MCEvoY, C. L. (1979). Encoding context and set size. Journal of Experimental Psychology: Human Learning \& Memory, 5, 292-314.

Nelson, D. L., \& McEvoy, C. L. (2000). What is this thing called frequency? Memory \& Cognition, 28, 509-522.

Nelson, D. L., McEvoy, C. L.. \& Dennis, S. (2000). What is free association and what does it measure? Memory \& Cognition, 28, 887 899.

Nelson, D. L., McEvoy, C. L., \& Schreiber, T. A. (1990). Encoding context and retrieval conditions as determinants of the effects of nat- ural category size. Journal of Experimental Psychology: Learning, Memory, \& Cognition, 16, 34-41.

Nelson, D. L., \& McEvoy, C. L., \& Schreiber, T. A. (1999). The University of South Florida word association, rhyme and word fragment norms [WWW document]. Available: http://www.usf.edu/ FreeAssociation/

Nelson, D. L., McKinney, V. M., Gee, N. R., \& Janczura, G. A. (1998). Interpreting the influence of implicitly activated memories on recall and recognition. Psychological Review, 105, 299-324.

Nelson, D. L., \& SChreiBer, T. A. (1992). Word concreteness and word structure as independent determinants of recall. Journal of Memory \& Language, 31, 237-260.

Nel.son, D. L., Schreiber, T. A., \& Holley, P. E. (1992). The retrieval of controlled and automatic aspects of meaning on direct and indirect tests. Memory \& Cognition, 20, 671-684.

Nelson, D. L., SChreiber, T. A., \& McEvoy, C. L. (1992). Processing implicit and explicit representations. Psychological Review, 99, 322-348.

Nelson, D. L., Schreiber, T. A., \& XU, J. (1999). Cue set size effects: Sampling activated associates or cross-target interference? Memory \& Cognition, 27, 465-477.

NeLsON, D. L., \& XU, J. (1995). Effects of implicit memory on explicit recall: Set size and word frequency effects. Psychological Research, 57, 203-214.

Paivio, A., Yuille, J. C., \& Madigan, S. (1968). Concreteness, imagery, and meaningfulness values for 925 nouns. Journal of Experimental Psychology Monograph Supplement, 76 (1, Pt. 2), 1-25.

RaAiJmakers, J. G., \& Shiffrin, R. M. (1981). Search of associative memory. Psychological Review, 88, 93-134.

Roediger, H. L., III, \& MCDERMOTT, K. B. (1993). Implicit memory in normal human subjects. In F. Boller \& J. Grafman (Eds.), Handbook of neuropsychology (Vol. 8, pp. 63-131). Amsterdam: Elsevier.

Rubin, D. C., \& Friendly, M. (1986). Predicting which words get recalled: Measures of free recall, availability, goodness, emotionality, and pronunciability for 925 nouns. Memory \& Cognition, 14, 79-94.

Tulving, E., \& Thomson, D. M. (1973). Encoding specificity and retrieval processes in episodic memory. Psychological Review, 80, 352-373.

\section{NOTES}

1. The true correlation between free association and cued recall was computed as

$$
r_{T x T y}=\frac{r_{x y}}{\sqrt{r_{x x} \sqrt{r_{y y}}}}=\frac{.56}{\sqrt{.89 \sqrt{ } .73}}=.70 .
$$

2. For people interested in checking the present models or in creating new models, the cued recall database used for this paper is available on disk on request and can be found on the Web: (http://www.usf.edu/ $\sim$ nelson/CuedRecallDatabase).

\section{APPENDIX A \\ An Example Calculation of Equation 1 for the Word DinNER}

1. $n \times n$ association matrix for the word DINNER, showing its associates and their interconnection strengths as indexed by free association norms. These values are used to calculate Equation 1.

\begin{tabular}{lcccccc}
\hline DINNER & Dinner & Supper & Eat & Lunch & Food & Meal \\
\hline Dinner & 1.00 & .54 & .11 & .10 & .09 & .09 \\
Supper & .55 & - & .02 & .03 & .17 & .01 \\
Eat & & & - & & .40 & .02 \\
Lunch & .27 & .02 & .08 & - & .21 & .06 \\
Food & & & .41 & .01 & - & .02 \\
Meal & .21 & .06 & .06 & .06 & .49 & - \\
\hline
\end{tabular}

2. Calculation of the net activation strength of the target word DINNER, using Equation 1, as defined by 
APPENDIX A (Continued)

$$
\begin{aligned}
& S\left(T_{i}\right)=a_{i}+\sum_{j}^{n} a_{j} r_{i j}, \\
& \text { where } r_{i j}=w_{i j}+\sum_{k}^{n} a_{k} r_{j k},
\end{aligned}
$$

with $a_{i}=1$, and the connection strengths of Section 1 . In this example, $S\left(T_{i}\right)$ is affected by three resonant loops to the target involving supper, lunch, and meal. The associates eat and food have been excluded because they show no connection to DINNER in the norms. Also note that calculations reflecting the influence of other associates on any given associate $a_{k}$ are shown below in Section 3 . For example, connections from other associates in

\begin{tabular}{|c|c|c|c|c|c|}
\hline Loop & $a_{k} \times r_{j k}$ & $w_{i j}$ & $r_{i j}$ & $a_{j}$ & $a_{j} \times r_{i j}$ \\
\hline \multicolumn{6}{|l|}{ Supper } \\
\hline Dinner-to-lunch $(.10) \times$ lunch-to-supper $(.02+.0225)$ & .0043 & & & & \\
\hline \multirow[t]{2}{*}{ Dinner-to-meal $(.09) \times$ meal-to-supper $(.06+.0154)$} & .0068 & & & & \\
\hline & .0111 & .55 & .5611 & .54 & 3030 \\
\hline \multicolumn{6}{|l|}{ Lunch } \\
\hline Dinner-to-supper $(.54) \times$ supper-to-lunch $(.03+.0074)$ & 0202 & & & & \\
\hline Dinner-to-meal $(.09) \times$ meal-to-lunch $(.06+.0154)$ & .0068 & & & & \\
\hline Dinner-to-food $(.09) \times$ food-to-lunch $(.01+.2009)$ & .0189 & & & & \\
\hline & .0459 & .27 & .3159 & .10 & .0316 \\
\hline \multicolumn{6}{|l|}{ Meal } \\
\hline Dinner-to-supper $(.54) \times$ supper-to- & .0094 & & & & \\
\hline Dinner-to-eat $(.11) \times$ eat-to-meal $(.02+.0611)$ & .0089 & & & & \\
\hline Dinner-to-lunch $(.10) \times$ lunch-to-meal $(.06+.0225)$ & .0083 & & & & \\
\hline Dinner-to-food $(.09) \times$ food-to-meal $(.02+.2009)$ & .0198 & & & & \\
\hline $\begin{array}{l}\text { Activation fro } \\
\text { Net activation }\end{array}$ & .0464 & .21 & .2564 & .09 & $\begin{array}{r}.0231 \\
.3577 \\
1.3577\end{array}$ \\
\hline
\end{tabular}
the set to the associate lunch sum to.0225.

3. Connections from other associates in the set to each associate that add to its activation are given by

$$
S\left(A_{j}\right)=\sum_{k}^{n} a_{k} r_{j k}
$$

(e.g., $S[$ Lunch] $=.0225$ ).

\begin{tabular}{ll}
\hline & $S\left(A_{j}\right)=\sum_{k}^{n} a_{k} r_{j k}$ \\
\hline Let & \\
$\quad$ Dinner & .0162 \\
Dinner-to-supper $(.54) \times$ supper-to-lunch $(.03)$ & .0054 \\
Dinner-to-food $(.09) \times$ meal-to-lunch $(.06)$ & $\underline{.0009}$ \\
& .0225 \\
Meal & \\
$\quad$ Dinner-to-supper $(.54) \times$ supper-to-lunch $(.01)$ & .0054 \\
Dinner-to-eat $(.11) \times$ eat-to-meal $(.02)$ & .0022 \\
Dinner-to-lunch $(.10) \times$ lunch-to-meal $(.06)$ & .0060 \\
Dinner-to-food $(.09) \times$ food-to-meal $(.02)$ & .0018 \\
& .0154 \\
Supper & .0020 \\
Dinner-to-lunch $(.10) \times$ lunch-to-supper $(.02)$ & .0054 \\
Dinner-to-meal $(.09) \times$ meal-to-supper $(.06)$ & .0074 \\
& \\
Food & .0918 \\
Dinner-to-supper $(.54) \times$ supper-to-food $(.17)$ & .0440 \\
Dinner-to-eat $(.11) \times$ eat-to-food $(.40)$ & .0210 \\
Dinner-to-lunch $(.10) \times$ lunch-to-food $(.21)$ & .0441 \\
Dinner-to-meal $(.09) \times$ meal-to-food $(.49)$ & .2009 \\
& \\
Eat & .0108 \\
Dinner-to-supper $(.54) \times$ supper-to-eat $(.02)$ & .0080 \\
Dinner-to-lunch $(.10) \times$ lunch-to-eat $(.08)$ & .0369 \\
Dinner-to-food $(.09) \times$ food-to-eat $(.41)$ & .0054 \\
Dinner-to-meal $(.09) \times$ meal-to-eat $(.06)$ & .0611 \\
\hline
\end{tabular}




\section{APPENDIX B \\ An Example Calculation Involving the Target Word DINNER and the Test Cue LuNCH, Using Equation $1 A$ and Equation 2 from PIER 2}

1. Associative matrix for DINNER and calculation of Equation 1A. DINNER is assigned a nominal value of 1.00 because it is presented, and the remaining values are obtained from free association norms.

\begin{tabular}{|c|c|c|c|c|c|c|}
\hline inner & 1.00 & .54 & .11 & .10 & .09 & .09 \\
\hline upper & .55 & - & .02 & .03 & .17 & .01 \\
\hline at & & & & & .4 & \\
\hline unch & .27 & .02 & .08 & - & .21 & .06 \\
\hline od & & & .41 & .01 & - & .02 \\
\hline Teal & .21 & .06 & .06 & .06 & .4 & - \\
\hline$S\left(A_{i}\right)$ & 2.03 & .62 & .68 & .20 & 1.36 & .20 \\
\hline
\end{tabular}

2. Calculation of Equation 2 with LUNCH as the test cue and DINNER as the target. $S=$ shared associate; $M=$ mediator. The results of Equation $1 \mathrm{~A}$ for the target, the test cue, and each of its shared associates are folded into the top row of the retrieval matrix to compute PIER 2's Equation 2. The calculation of Equation 2 assumes that prior study of the target affects only its self-strength and the strength of the links between it and each of its associates. In other words, it assumes that links emanating from the test cue are unaffected by the target presentation. Links between the test cue and the target and between the test cue and each shared associate and each mediator are left at baseline levels estimated by free association norms. This case also applies to the mediator-to-target connections (e.g., the supper-to-dinner [.55] and meal-to-dinner [.21] links). With this simplifying assumption, PIER 2's Equation 2 is calculated as

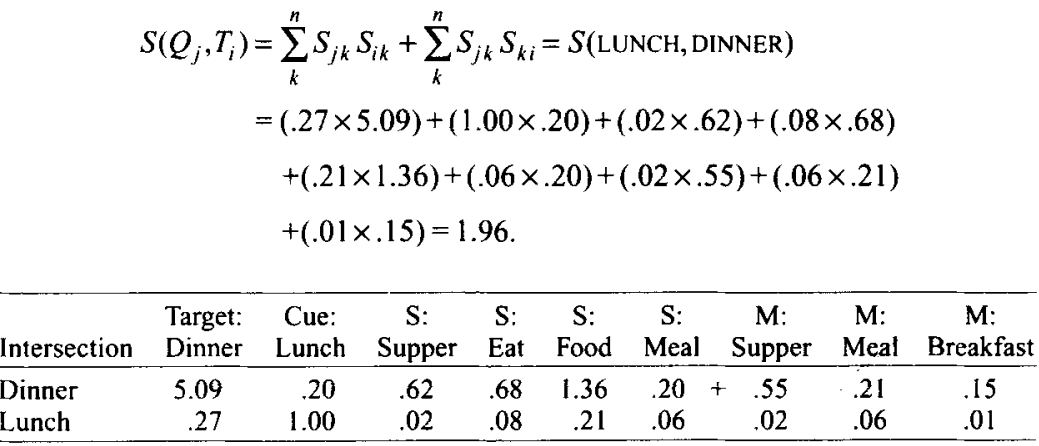

